\title{
WAVELETS IN WANDERING SUBSPACES
}

\author{
T. N. T. GOODMAN, S. L. LEE, AND W. S. TANG
}

\begin{abstract}
Mallat's construction, via a multiresolution approximation, of orthonormal wavelets generated by a single function is extended to wavelets generated by a finite set of functions. The connection between multiresolution approximation and the concept of wandering subspaces of unitary operators in Hilbert space is exploited in the general setting. An example of multiresolution approximation generated by cardinal Hermite $B$-splines is constructed.
\end{abstract}

\section{INTRODUCTION}

Wavelets are functions generated by translating and dilating a function or a finite set of functions. They are useful in many areas of mathematics and theoretical physics $[1,2]$ and also in practical applications such as image and signal processing $[13,14]$. Orthonormal bases of wavelets generated by one function have been constructed for various function spaces $[11,15,16]$. Recently, Mallat [12] has unified the construction of these bases for $L^{2}(\mathbf{R})$ via the multiresolution approximation which is defined to be a sequence of closed subspaces $\left(V_{m}\right)_{m \in \mathbf{Z}}$ of $L^{2}(\mathbf{R})$ satisfying the following properties:

$$
\begin{gathered}
V_{m} \subset V_{m+1}, \quad m \in \mathbf{Z}, \\
\bigcup_{m \in \mathbf{Z}} V_{m} \text { is dense in } L^{2}(\mathbf{R}) \quad \text { and } \\
f \in V_{m} \Leftrightarrow D_{2} f \in V_{m+1}, \quad m \in \mathbf{Z} V_{m}=\{0\},
\end{gathered}
$$

where $D_{a} f(x):=f(a x), x \in \mathbf{R}$, for any positive number $a$,

$$
f \in V_{m} \Leftrightarrow T_{2^{-m_{n}}} f \in V_{m}, \quad(m, n) \in \mathbf{Z}^{2},
$$

where $T_{\tau}(x):=f(x-\tau), x \in \mathbf{R}$, for any $\tau \in \mathbf{R}$,

there exists an isomorphism $\mathscr{I}: V_{0} \rightarrow l^{2}(\mathbf{Z})$ which commutes with the action of $\mathbf{Z}$.

For a multiresolution approximation $\left(V_{m}\right)_{m \in \mathbf{Z}}$, Mallat [12] has shown the existence of $\phi \in V_{0}$ such that $\left(T_{n} \phi\right)_{n \in \mathbf{Z}}$ is an orthonormal basis of $V_{0}$. Furthermore, if

$$
\phi_{m, n}(x):=\sqrt{2^{m}} \phi\left(2^{m} x-n\right), \quad(m, n) \in \mathbf{Z}^{2},
$$

Received by the editors April 30, 1991.

1980 Mathematics Subject Classification (1985 Revision). Primary 41A15, 42C15, 47B37.

Key words and phrases. Wavelets, Hilbert space, wandering subspaces, unitary operators, Hermite $B$-splines. 
then for each $m \in \mathbf{Z},\left(\phi_{m, n}\right)_{n \in \mathbf{Z}}$ is an orthonormal basis of $V_{m}$. Let $W_{m}$ be the orthogonal complement of $V_{m}$ in $V_{m+1}$. Mallat has also shown the existence of $\psi \in W_{0}$ such that $\left(T_{n} \psi\right)_{n \in Z}$ is an orthonormal basis of $W_{0}$, and if

$$
\psi_{m, n}(x):=\sqrt{2^{m}} \psi\left(2^{m} x-n\right), \quad(m, n) \in \mathbf{Z}^{2},
$$

then $\left(\psi_{m, n}\right)_{n \in \mathbf{Z}}$ is a complete orthonormal set in $W_{m}$.

Properties (1.1) and (1.2) of the multiresolution approximation imply that

$$
W_{j} \perp W_{k}, \quad j \neq k,
$$

and

$$
L^{2}(\mathbf{R})=\sum_{m \in \mathbf{Z}} \oplus W_{m}
$$

Since $\left(\psi_{m, n}\right)_{n \in \mathbf{Z}}$ is an orthonormal basis of $W_{m}$, it follows that $\left(\psi_{m, n}\right)_{(m, n) \in \mathbf{Z}^{2}}$ is an orthonormal basis of $L^{2}(\mathbf{R})$.

Our object is to extend Mallat's results to wavelets generated by a finite number of functions. In this connection we observe the relationship between wavelets and the concept of wandering subspaces in operator theory (see Halmos [6, Problem 155], and Robertson [17]). This provides a general setting to wavelets in Hilbert space where the translation and dilation operators are replaced by unitary operators. We prove the existence of orthonormal wavelet bases for $V_{m}$ generated by a finite number of vectors, and using a result of Robertson [17], we also prove the existence of bases of orthonormal wavelets for $W_{m}$ and derive other results which are reminiscent of multiresolution approximation. This is done in $\S 2$. A more detailed analysis of the corresponding results in $L^{2}(\mathbf{R})$ is given in $\S 3$. An example of multiresolution approximation generated by cardinal Hermite $B$-splines (see Schoenberg [18, Chapter 5]) is constructed in $\S 4$. In $\S 5$ we construct compactly supported Hermite spline wavelets which extends the results of Chui and Wang [4].

\section{EXISTENCE OF ORTHONORMAL WAVELETS AND WANDERING SUBSPACES}

Let $T$ be a unitary operator on a complex Hilbert space $\mathscr{H}$. A subspace $\mathscr{S}$ of $\mathscr{H}$ is called a wandering subspace for $T$ if $T^{m}(\mathscr{S}) \perp T^{n}(\mathscr{S})$ for all $m \neq n$ (see $[6,17])$. Further, if $V=\sum_{n \in \mathbf{Z}} \oplus T^{n}(\mathscr{S})$, then we say that $\mathscr{S}$ is a complete wandering subspace of $V$ for $T$. We shall denote by $E$ the forward shift operator on $l^{2}(\mathbf{Z})$.

We begin by stating the Fuglede-Putnam Theorem in the form which will be needed later.

Lemma 2.1. Let $\mathscr{H}_{i}, i=1,2$, be complex Hilbert spaces, and $A_{i}: \mathscr{H}_{i} \rightarrow \mathscr{H}_{i}$, $i=1,2$, be normal operators. If $B: \mathscr{H}_{2} \rightarrow \mathscr{H}_{1}$, is a bounded operator such that $A_{1} B=B A_{2}$, then $A_{1}^{*} B=B A_{2}^{*}$. If in addition $B$ is invertible, then there exists a unitary operator $U: H_{2} \rightarrow H_{1}$ such that $A_{1} U=U A_{2}$.

When $\mathscr{H}_{1}=\mathscr{H}_{2}$, a proof of Fuglede-Putnam Theorem can be found in [6, Solution 192]. The proof with obvious modifications works for the general case.

Theorem 2.1. Let $V_{0}$ be a closed subspace of a complex Hilbert space $\mathscr{H}$, and $T$ a unitary operator on $\mathscr{H}$ such that

$$
T V_{0}=V_{0} \text {. }
$$


Suppose that there exists a bounded invertible operator $J: V_{0} \rightarrow l^{2}(\mathbf{Z})$ such that

$$
J T=E^{r} J
$$

for some $r \in \mathbf{Z} \backslash\{0\}$, and a unitary operator $D$ on $\mathscr{H}$ such that

$$
V_{0} \subset D V_{0},
$$

and

$$
T D=D T^{k}
$$

for some $k \in \mathbf{Z},|k|>1$. Let

$$
V_{m}:=D^{m} V_{0}, \quad m \in \mathbf{Z} .
$$

Then the following results hold:

(1) For every $m \in \mathbf{Z}, V_{m} \subset V_{m+1}$.

(2) There exist $\phi_{j} \in \mathscr{H}, 1 \leq j \leq|r|$, such that

$$
\left\{T^{n} \phi_{j}: n \in \mathbf{Z}, 1 \leq j \leq|r|\right\}
$$

is an orthonormal basis of $V_{0}$.

(3) The set

$$
\left\{T^{n} D T^{l} \phi_{j}: n \in \mathbf{Z}, 0 \leq l \leq|k|-1, \quad 1 \leq j \leq|r|\right\}
$$

is an orthonormal basis of $V_{1}$.

(4) There exist $\psi_{j}, 1 \leq j \leq|r|(|k|-1)$, such that

$$
\left\{T^{n} \psi_{j}: n \in \mathbf{Z}, \quad 1 \leq j \leq|r|(|k|-1)\right\}
$$

is an orthonormal basis of the orthogonal complement $W_{0}$ of $V_{0}$ in $V_{1}$.

(5) The collection

$$
\left\{D^{m} T^{n} \psi_{j}:(m, n) \in \mathbf{Z}^{2}, 1 \leq j \leq|r|(|k|-1)\right\}
$$

is an orthonormal basis of $\overline{\bigcup_{m \in \mathbf{Z}} V_{m}} \ominus \bigcap_{m \in \mathbf{Z}} V_{m}$.

Remark 1. The result (2) of Theorem 2.1 is equivalent to the existence of a complete $|r|$-dimensional wandering subspace of $V_{0}$ for $T$. Similarly, (4) is equivalent to the existence of a complete $|r|(|k|-1)$-dimensional wandering subspace of $W_{0}$ for $T$.

Remark 2. For a nonzero integer $r$, condition (2.2) is equivalent to the existence of a bounded invertible operator $\widetilde{J}: V_{0} \rightarrow l^{2}(\mathbf{Z})^{|r|}$ such that $\widetilde{J} T=\widetilde{E} \widetilde{J}$, where $\widetilde{E}\left(s_{1}, \ldots, s_{|r|}\right)=\left(E^{\varepsilon} s_{1}, \ldots, E^{\varepsilon} s_{|r|}\right),\left(s_{1}, \ldots, s_{|r|}\right) \in l^{2}(\mathbf{Z})^{|r|}, \varepsilon:=r /|r|$. Remark 3. If $\mathscr{\ell}=L^{2}(\mathbf{R})$, and $T=T_{1}$ and $D=D_{2}$ are the translation and dilation operators respectively on $L^{2}(\mathbf{R})$, then $T$ and $D$ satisfy condition (2.4) with $k=2$. In this case Theorem 2.1 , with $r=1$, reduces to the results of Mallat [12] on the existence of orthonormal wavelet bases for $V_{m}$ and its orthogonal complement $W_{m}$ in $V_{m+1}$ corresponding to a multiresolution approximation $\left(V_{m}\right)_{m \in \mathbf{Z}}$ of $L^{2}(\mathbf{R})$.

Proof of Theorem 2.1. It is clear from the definition that $V_{m} \subset V_{m+1}, m \in \mathbf{Z}$.

Let $e_{j} \in l^{2}(\mathbf{Z})$ such that $e_{j}(n)=\delta_{j n}, j, n \in \mathbf{Z}$. Then, $\left\{\left(E^{r}\right)^{n} e_{j}: n \in \mathbf{Z}\right.$, $1 \leq j \leq|r|\}$ is an orthonormal basis of $l^{2}(\mathbf{Z})$. Since $T$ and $E^{r}$ are normal (indeed unitary) operators on $V_{0}$ and $l^{2}(\mathbf{Z})$ respectively, and they are similar by 
(2.2), it follows from Fuglede-Putnam Theorem (Lemma 2.1), that there exists a unitary operator $U: V_{0} \rightarrow l^{2}(\mathbf{Z})$ such that $U T=E^{r} U$. If $\phi_{j}:=U^{-1} e_{j}$, $1 \leq j \leq|r|$, then $\left\{T^{n} \phi_{j}: n \in \mathbf{Z}, 1 \leq j \leq|r|\right\}$ is an orthonormal basis of $V_{0}$. Hence $\mathscr{X}:=\operatorname{span}\left\{\phi_{j}: 1 \leq j \leq|r|\right\}$ is an $|r|$-dimensional complete wandering subspace of $V_{0}$ for $T$. This proves (2).

Since $D$ is a unitary operator on $\mathscr{H}$, and $V_{m}:=D^{m} V_{0},\left\{D^{m} T^{n} \phi_{j}: n \in \mathbf{Z}\right.$, $1 \leq j \leq|r|\}$ is an orthonormal basis of $V_{m}$. In particular, since $T^{n} D=D T^{n k}$, $n \in \mathbf{Z}$, the result (3) follows.

Let $\mathscr{Y}:=\operatorname{span}\left\{D T^{l} \phi_{j}: 0 \leq l \leq|k|-1,1 \leq j \leq|r|\right\}$. Then by (3), $\mathscr{Y}$ is an $|r k|$-dimensional complete wandering subspace of $V_{1}$ for $T$. By (2.3),

$$
\sum_{n \in \mathbf{Z}} \oplus T^{n}(\mathscr{X}) \subset \sum_{n \in \mathbf{Z}} \oplus T^{n}(\mathscr{Y}) .
$$

By a theorem of Robertson [17, Theorem 2, p. 235], there exist vectors $\psi_{j}$, $1 \leq j \leq|r|(|k|-1)$, such that $\left\{T^{n} \psi_{j}: n \in \mathbf{Z}, 1 \leq j \leq|r|(|k|-1)\right\}$ is an orthonormal basis of the orthogonal complement $W_{0}$ of $V_{0}$ in $V_{1}$.

Finally, letting $W_{m}$ be the orthogonal complement of $V_{m}$ in $V_{m+1}$, a standard argument shows that $W_{m}=D^{m}\left(W_{0}\right), m \in \mathbf{Z}$, and

$$
\overline{\bigcup_{m \in \mathbf{Z}} V_{m}} \ominus \bigcap_{m \in \mathbf{Z}} V_{m}=\sum_{m \in \mathbf{Z}} \oplus W_{m} \text {. }
$$

Hence, for each $m,\left\{D^{m} T^{n} \psi_{j}: n \in \mathbf{Z}, 1 \leq j \leq|r|(|k|-1)\right\}$ is an orthonormal basis of $W_{m}$ and the collection $\left\{D^{m} T^{n} \psi_{j}:(m, n) \in \mathbf{Z}^{2}, 1 \leq j \leq|r|(|k|-1)\right\}$ is an orthonormal basis of $\overline{\bigcup_{m \in \mathbf{Z}} V_{m}} \ominus \bigcap_{m \in \mathbf{Z}} V_{m}$.

Remark 4. In Theorem 2.1, it was assumed that (2.4) holds with $|k| \neq 1$. Suppose that it holds with $|k|=1$, and all the other hypotheses of the theorem are satisfied. Then as in the proof of the theorem, $\mathscr{X}$ and $\mathscr{Y}$ are both $|r|$ dimensional wandering subspaces for $T$, and

$$
V_{0}=\sum_{n \in \mathbf{Z}} \oplus T^{n}(\mathscr{X}) \subset \sum_{n \in \mathbf{Z}} \oplus T^{n}(\mathscr{Y})=V_{1} .
$$

By Theorem 1 of [17], this implies that $V_{0}=V_{1}$. Hence $W_{0}=\{0\}$, and $V_{m}=V_{0}$ for all $m \in \mathbf{Z}$.

3. WAVELETS GENERATED BY A FINITE SET OF FUNCTIONS IN $L^{2}(\mathbf{R})$

Let $r$ be a positive integer and

$$
l^{2}(\mathbf{Z})^{r}:=\left\{\left(s_{1}, \ldots, s_{r}\right): s_{j} \in l^{2}(\mathbf{Z}), j=1, \ldots, r\right\} .
$$

For $s=\left(s_{1}, \ldots, s_{r}\right) \in l^{2}(\mathbf{Z})^{r}$, its norm is given by

$$
\|s\|:=\left(\sum_{j=1}^{r}\left\|s_{j}\right\|^{2}\right)^{1 / 2} .
$$

The space of $2 \pi$-periodic square integrable functions and the space of $2 \pi$ periodic continuous functions will be denoted by $\widetilde{L}^{2}(0,2 \pi)$ and $\widetilde{C}(0,2 \pi)$ respectively. The Fourier transform of $f \in L^{2}(\mathbf{R})$ will be denoted by $\hat{f}$. 
Let $g_{j} \in L^{2}(\mathbf{R}), j=1, \ldots, r$, such that $\sum_{n \in \mathbf{Z}} s(n) T_{n} g_{j} \in L^{2}(\mathbf{R})$, for all $s=(s(n))_{n \in \mathbf{Z}} \in l^{2}(\mathbf{Z})$. Then $\sum_{n \in \mathbf{Z}}\left|\hat{g}_{j}(u+2 \pi n)\right|^{2}$ is integrable, and for almost all $u \in \mathbf{R},\left(\hat{g}_{j}(u+2 \pi n)\right)_{n \in \mathbf{Z}} \in l^{2}(\mathbf{Z})$. Let

$$
\hat{g}_{i j}(u):=\sum_{n \in \mathbf{Z}} \hat{g}_{i}(u+2 \pi n) \overline{\hat{g}_{j}(u+2 \pi n)}, \quad i, j=1, \ldots, r,
$$

and

$$
\widehat{G}(u):=\left(\hat{g}_{i j}(u)\right)_{i, j=1}^{r} .
$$

Then $\widehat{G}$ is Hermitian, and therefore there is a unitary matrix $U$ such that

$$
\widehat{G}=U^{*} D U,
$$

where $U^{*}$ denotes the conjugate transpose of $U$,

$$
D(u):=\operatorname{diag}\left(\lambda_{1}(u), \ldots, \lambda_{r}(u)\right),
$$

and $\lambda_{j}$ are the eigenvalues of $\widehat{G}$.

Theorem 3.1. Let $K: l^{2}(\mathbf{Z})^{r} \rightarrow L^{2}(\mathbf{R})$ be defined by

$$
K(s):=\sum_{j=1}^{r} \sum_{n \in \mathbf{Z}} s_{j}(n) T_{n} g_{j}, \quad s:=\left(s_{1}, \ldots, s_{r}\right) \in l^{2}(\mathbf{Z})^{r} .
$$

Then $K$ is an isomorphism onto a subspace of $L^{2}(\mathbf{R})$ if and only if there exist positive constants $A$ and $B$ such that

$$
A \leq \lambda_{j}(u) \leq B, \quad j=1, \ldots, r \text {, almost everywhere. }
$$

Furthermore, $\left\{T_{n} g_{j}: n \in \mathbf{Z}, j=1, \ldots, r\right\}$ is an orthonormal subset of $L^{2}(\mathbf{R})$ if and only if $K$ is an isometry, and these hold if and only if the matrix $\widehat{G}(u)$ is the identity for almost all $u$.

Proof. For $s=\left(s_{1}, \ldots, s_{r}\right) \in l^{2}(\mathbf{Z})^{r}$,

$$
\widehat{K(s)}(u)=\sum_{j=1}^{r} h_{j}(u) g_{j}(u)
$$

where

$$
h_{j} \in \widetilde{L}^{2}(0,2 \pi) \quad \text { with }\left\|h_{j}\right\|=\left\|s_{j}\right\| .
$$

Therefore,

$$
\|\widehat{K(s)}\|^{2}=\int_{-\infty}^{\infty} \sum_{i=1}^{r} \sum_{j=1}^{r} h_{i}(u) \overline{h_{j}(u)} \hat{g}_{i}(u) \overline{\hat{g}_{j}(u)} d u .
$$

Decomposing the integral over the intervals $[2 \pi n, 2 \pi(n+1)), n \in \mathbf{Z}$, and using (3.1) leads to

$$
\|\widehat{K(s)}\|^{2}=\int_{0}^{2 \pi} \sum_{i=1}^{r} \sum_{j=1}^{r} h_{i}(u) \overline{h_{j}(u)} \hat{g}_{i j}(u) d u .
$$

The integrand of the last integral is a quadratic form. Using the Parseval identity and letting $H(u):=\left(h_{1}(u), \ldots, h_{r}(u)\right)^{*}$, it can be written as

$$
\|K(s)\|^{2}=\frac{1}{2 \pi} \int_{0}^{2 \pi} H^{*} \widehat{G} H(u) d u .
$$


By (3.3), $H^{*} \widehat{G} H=Q^{*} D Q$, where

$$
Q=\left(q_{1}, \ldots, q_{r}\right)^{*}:=U H
$$

with

$$
\sum_{j=1}^{r}\left\|q_{j}\right\|^{2}=\sum_{j=1}^{r}\left\|h_{j}\right\|^{2}
$$

since $U$ is unitary. Therefore (3.7) becomes

$$
\|K(s)\|^{2}=\frac{1}{2 \pi} \int_{0}^{2 \pi} Q^{*} D Q(u) d u=\frac{1}{2 \pi} \int_{0}^{2 \pi} \sum_{j=1}^{r}\left|q_{j}(u)\right|^{2} \lambda_{j}(u) d u .
$$

Now, $K$ is an isomorphism if and only if there exist positive constants $A$ and $B$ such that

$$
A\|s\|^{2} \leq\|K(s)\|^{2} \leq B\|s\|^{2}, \quad s \in l^{2}(\mathbf{Z})^{r} .
$$

By (3.6) and (3.8), this is equivalent to

$$
\begin{aligned}
A \sum_{j=1}^{r} \int_{0}^{2 \pi}\left|q_{j}(u)\right|^{2} d u & \leq \sum_{j=1}^{r} \int_{0}^{2 \pi}\left|q_{j}(u)\right|^{2} \lambda_{j}(u) d u \\
& \leq B \sum_{j=1}^{r} \int_{0}^{2 \pi}\left|q_{j}(u)\right|^{2} d u
\end{aligned}
$$

for all $q_{j} \in L^{2}(0,2 \pi), j=1, \ldots, r$, which in turn is equivalent to (3.5). tions.

The last assertion of the theorem is an easy consequence of the above rela-

A function $f \in L^{2}(\mathbf{R})$ is said to be regular if $\hat{f}$ is continuous and satisfies $\hat{f}(u)=O\left(u^{-1}\right)$ as $|u| \rightarrow \infty$.

Corollary 3.1. Suppose that $g_{j}, j=1, \ldots, r$, are regular. Then $K$ is an isomorphism onto a subspace of $L^{2}(\mathbf{R})$ if and only if $\widehat{G}(u)$ is positive definite for all $u$.

Proof. The assumption that $g_{j}, j=1, \ldots, r$, are regular implies that $\hat{g}_{i j}$, $i, j=1, \ldots, r$, are continuous. If $\widehat{G}(u)$ is positive definite for all $u$, its eigenvalues $\lambda_{j}(u)$ are positive for all $u$ and $j=1, \ldots, r$. Since $\lambda_{j}$ are $2 \pi$ periodic and continuous, there exist positive constants $A$ and $B$ such that (3.5) hold for all $u$. The converse is immediate.

Theorem 3.2. Suppose that $g_{j}, j=1, \ldots, r$, are regular. Then $K$ is an isomorphism onto a subspace of $L^{2}(\mathbf{R})$ if and only if for each $u$, the infinite matrix

$$
M(u):=\left(\hat{g}_{i}(u+2 \pi j)\right)_{i=1, j \in \mathbf{Z}}^{r}
$$

has rank $r$.

Proof. By (3.2) and (3.9), $\widehat{G}=M M^{*}$. For any $H=\left(h_{1}, \ldots, h_{r}\right)^{*} \in \mathbf{C}^{r}$,

$$
H^{*} \widehat{G} H=\left(H^{*} M\right)\left(H^{*} M\right)^{*}=\sum_{j \in \mathbf{Z}}\left|\sum_{i=1}^{r} h_{i} \hat{g}_{i}(u+2 \pi j)\right|^{2} \text {. }
$$


Therefore, for any $H \neq 0, H^{*} \widehat{G} H>0$ if and only if $\sum_{i=1}^{r} h_{i} \hat{g}_{i}(u+2 \pi j)$ for some $j$, if and only if $\operatorname{rank}(M)=r$. $C$.

In the next theorem, the notation $C_{j}$ means the $j$ th column of the matrix

Theorem 3.3. Suppose that $K$ is an isomorphism. Let

$$
D^{1 / 2}(u):=\operatorname{diag}\left(\lambda_{1}(u)^{1 / 2}, \ldots, \lambda_{r}(u)^{1 / 2}\right),
$$

and

$$
\left(\hat{\phi}_{1}(u), \ldots, \hat{\phi}_{r}(u)\right)^{T}:=\left(\left(U^{*} D^{1 / 2}\right)^{-1} M\right)_{0} .
$$

Then $\left\{T_{n} \phi_{j}: n \in \mathbf{Z}, j=1, \ldots, r\right\}$ is an orthonormal set.

Proof. Since $U^{*}$ and $D^{1 / 2}$ are $2 \pi$-periodic, by (3.10)

$$
\left(\hat{\phi}_{i}(u+2 \pi j)\right)_{i=1, j \in \mathbf{Z}}^{r}=\left(U^{*} D^{1 / 2}\right)^{-1} M .
$$

Let

$$
\hat{\phi}_{i j}(u):=\sum_{n \in \mathbf{Z}} \hat{\phi}_{i}(u+2 \pi n) \overline{\hat{\phi}_{j}(u+2 \pi n)},
$$

and $\hat{\boldsymbol{\Phi}}:=\left(\hat{\phi}_{i j}\right)_{i, j=1}^{r}$. Then

$$
\hat{\boldsymbol{\Phi}}=\left(U^{*} D^{1 / 2}\right)^{-1} M M^{*}\left(D^{1 / 2} U\right)^{-1} .
$$

Since $M M^{*}=\widehat{G}=\left(U^{*} D^{1 / 2}\right)\left(D^{1 / 2} U\right)$, it follows that $\hat{\Phi}(u)$ equals the identity matrix for almost all $u$. By Theorem 3.1, the map $K_{1}: l^{2}(\mathbf{Z})^{r} \rightarrow L^{2}(\mathbf{R})$ defined by

$$
K_{1}(s):=\sum_{j=1}^{r} \sum_{n \in \mathbf{Z}} s_{j}(n) T_{n} \phi_{j}, \quad s \in l^{2}(\mathbf{Z})^{r},
$$

is an isometry onto a subspace of $L^{2}(\mathbf{R})$. Therefore $\left\{T_{n} \phi_{j}: n \in \mathbf{Z}, j=\right.$ $1, \ldots, r\}$, is an orthonormal set.

Remark 5. Suppose that $K$ is an isomorphism onto a subspace $V_{0}$ of $L^{2}(\mathbf{R})$. Since $T_{1} V_{0}=V_{0}$ and $T_{1} K=K E^{r}$, Theorem 2.1 also yields the existence of $\phi_{j}, j=1, \ldots, r$, such that $\left\{T_{n} \phi_{j}: n \in \mathbf{Z}, j=1, \ldots, r\right\}$ is an orthonormal basis of $V_{0}$.

Equation (3.10) is more of theoretical interest since the eigenvalues and eigenvectors of $\widehat{G}$ are, in general, difficult to compute. We shall now give an inductive construction of the orthonormal wavelets which could be more useful in practice. Suppose that $\left\{\phi_{j}: 1 \leq j \leq r-1\right\}$, is a set of regular functions in $L^{2}(\mathbf{R})$ such that $\left\{T_{n} \phi_{j}: n \in \mathbf{Z}, 1 \leq j \leq r-1\right\}$ is an orthonormal set. Then by Theorem 3.1 the mapping $K_{1}: l^{2}(\mathbf{Z})^{r-1} \rightarrow L^{2}(\mathbf{R})$ defined by (3.12) with $r-1$ instead of $r$, is an isometry onto a subspace of $L^{2}(\mathbf{R})$, and $\hat{\boldsymbol{\Phi}}:=\left(\hat{\phi}_{i j}\right)_{i, j=1}^{r-1}$, where $\hat{\phi}_{i j}$ are defined in (3.11), is the identity matrix. 
Theorem 3.4. Suppose $g \in L^{2}(\mathbf{R})$ is regular. Then the map $K: l^{2}(\mathbf{Z})^{r} \rightarrow L^{2}(\mathbf{R})$, such that

$$
K(s):=\sum_{j=1}^{r-1} \sum_{n \in \mathbf{Z}} s_{j}(n) T_{n} \phi_{j}+\sum_{n \in \mathbf{Z}} s_{r}(n) T_{n} g,
$$

is an isomorphism onto a subspace $V_{0}$ of $L^{2}(\mathbf{R})$ if and only if for all $u \in \mathbf{R}$,

$$
|\langle\hat{g}, \hat{g}\rangle(u)|>\sum_{j=1}^{r-1}\left|\left\langle\hat{g}, \hat{\phi}_{j}\right\rangle(u)\right|^{2},
$$

where

$$
\langle\hat{g}, \hat{g}\rangle(u):=\sum_{n \in \mathbf{Z}}|\hat{g}(u+2 \pi n)|^{2},
$$

and

$$
\left\langle\hat{g}, \hat{\phi}_{j}\right\rangle(u):=\sum_{n \in \mathbf{Z}} \hat{g}(u+2 \pi n) \overline{\hat{\phi}_{j}(u+2 \pi n)} .
$$

Further, if (3.14) holds and

$$
\begin{aligned}
\tau(u) & :=\left(\langle\hat{g}, \hat{g}\rangle(u)-\sum_{j=1}^{r-1}\left|\left\langle\hat{g}, \hat{\phi}_{j}\right\rangle(u)\right|^{2}\right)^{-1 / 2}, \\
t_{j}(u) & :=-\tau(u)\left\langle\hat{g}, \hat{\phi}_{j}\right\rangle(u), \quad j=1, \ldots, r-1,
\end{aligned}
$$

and

$$
\hat{\phi}_{r}:=\sum_{j=1}^{r-1} t_{j} \hat{\phi}_{j}+\tau \hat{g}
$$

then $\left\{T_{n} \phi_{j}: n \in \mathbf{Z}, j=1, \ldots, r\right\}$ is an orthonormal basis of $V_{0}$.

Proof. Let $g_{j}:=\phi_{j}, j=1, \ldots, r-1$, and $g_{r}:=g$. Then $\widehat{G}$ is a matrix of order $r$ whose last row is $\left(\hat{g}_{r 1}, \ldots, \hat{g}_{r r}\right)$ and last column is $\left(\hat{g}_{r 1}, \ldots, \hat{g}_{r r}\right)^{*}$, and the remaining submatrix is the identity. Clearly, $\widehat{G}$ is positive definite if and only if $|\widehat{G}|>0$ which is equivalent to (3.14).

If $(3.14)$ holds then

$$
V_{0}=\left\{f: f=\sum_{j=1}^{r-1} \sum_{n \in \mathbf{Z}} s_{j}(n) T_{n} \phi_{j}+\sum_{n \in \mathbf{Z}} s_{r}(n) T_{n} g\right\} .
$$

Now $f \in V_{0}$ if and only if there are unique $h_{j} \in \widetilde{L}^{2}(0,2 \pi), j=1, \ldots, r$, with

$$
\hat{f}=\sum_{j=1}^{r-1} h_{j} \hat{\phi}_{j}+h_{r} \hat{g}
$$

Therefore, we want to find $\tau, t_{1}, \ldots, t_{r-1}$ in $\widetilde{L}^{2}(0,2 \pi)$ such that, with

$$
\hat{\phi}_{r}:=\sum_{j=1}^{r-1} t_{j} \hat{\phi}_{j}+\tau \hat{g}
$$


$\left\{T_{n} \phi_{j}: n \in \mathbf{Z}, j=1, \ldots, r\right\}$ is an orthonormal set. This is equivalent to

$$
\left\langle\hat{\phi}_{r}, \hat{\phi}_{j}\right\rangle=\delta_{r, j}, \quad j=1, \ldots, r .
$$

Since $\left\langle\hat{\phi}_{i}, \hat{\phi}_{j}\right\rangle=\delta_{i j}, i, j=1, \ldots, r-1$, it follows from (3.16) that (3.17) is equivalent to

$$
t_{j}+\tau\left\langle\hat{g}, \hat{\phi}_{j}\right\rangle=0, \quad j=1, \ldots, r-1,
$$

and

$$
\sum_{j=1}^{r-1}\left|t_{j}\right|^{2}+|\tau|^{2}\langle\hat{g}, \hat{g}\rangle+\bar{\tau} \sum_{j=1}^{r-1} t_{j}\left\langle\hat{\phi}_{j}, \hat{g}\right\rangle+\tau \sum_{j=1}^{r-1} \bar{t}_{j}\left\langle\hat{g}, \hat{\phi}_{j}\right\rangle=1 .
$$

Substituting for $t_{1}, \ldots, t_{r-1}$ from (3.18) into (3.19) leads to

$$
|\tau|^{2}=\left(\langle\hat{g}, \hat{g}\rangle-\sum_{j=1}^{r-1}\left|\left\langle\hat{g}, \hat{\phi}_{j}\right\rangle\right|^{2}\right)^{-1}
$$

By (3.14), the function

$$
\tau(u):=\left(\langle\hat{g}, \hat{g}\rangle(u)-\sum_{j=1}^{r-1}\left|\left\langle\hat{g}, \hat{\phi}_{j}\right\rangle(u)\right|^{2}\right)^{-1 / 2}
$$

is $2 \pi$-periodic and continuous, and therefore belongs to $\widetilde{L}^{2}(0,2 \pi)$. We then define

$$
t_{j}(u):=-\tau(u)\left\langle\hat{g}, \hat{\phi}_{j}\right\rangle(u), \quad j=1, \ldots, r-1,
$$

which belong to $\widetilde{L}^{2}(0,2 \pi)$ and satisfy (3.18) and (3.19). So with $\phi_{r}$ defined by (3.16), (3.20) and (3.21), $\left\{T_{n} \phi_{j}: n \in \mathbf{Z}, j=1, \ldots, r\right\}$ is an orthonormal set in $V_{0}$.

We denote the Fourier transform operator by $\mathscr{F}$ and define $U: \mathscr{F} V_{0} \rightarrow \mathscr{F} V_{0}$ such that

$$
U \hat{f}:=\sum_{j=1}^{r-1} h_{j} \hat{\phi}_{j}=h_{r} \hat{\phi}_{r}, \quad \hat{f}=\sum_{j=1}^{r-1} h_{j} \hat{\phi}_{j}+h_{r} \hat{g} \in V_{0},
$$

where $h_{j} \in \widetilde{L}^{2}(0,2 \pi), j=1, \ldots, r$. By (3.16) we see that $U$ is an isomorphism of $\mathscr{F} V_{0}$ onto itself. Since $\left\{T_{n} \phi_{j}: n \in \mathbf{Z}, j=1, \ldots, r\right\}$ is orthonormal, it follows that the map $\widetilde{K}: l^{2}(\mathbf{Z})^{r} \rightarrow V_{0}$ such that

$$
\widetilde{K}(s):=\sum_{j=1}^{r} \sum_{n \in \mathbf{Z}} s_{j}(n) T_{n} \phi_{j}, \quad s=\left(s_{1}, \ldots, s_{r}\right) \in l^{2}(\mathbf{Z})^{r},
$$

is an isometry. But then $\widetilde{K}=\mathscr{F}^{-1} U \mathscr{F} K$, so that $\widetilde{K}$ maps $l^{2}(\mathbf{Z})^{r}$ onto $V_{0}$. Thus $\left\{T_{n} \phi_{j}: n \in \mathbf{Z}, j=1, \ldots, r\right\}$ is complete in $V_{0}$.

Now suppose that $\phi_{j} \in L^{2}(\mathbf{R}), j=1, \ldots, r$, such that $\left\{T_{n} \phi_{j}: n \in \mathbf{Z}\right.$, $j=1, \ldots, r\}$ is an orthonormal set, and let $V_{0}$ be its closed linear span in $L^{2}(\mathbf{R})$. Then

$$
V_{0}=\left\{f \in L^{2}(\mathbf{R}): \hat{f}=\sum_{j=1}^{r} \hat{\phi}_{j} h_{j}, h_{j} \in \widetilde{L}^{2}(0,2 \pi)\right\} .
$$


Let $V_{1}:=D_{2} V_{0}$. Then

$$
V_{1}=\left\{f \in L^{2}(\mathbf{R}): \hat{f}(2 u)=\sum_{j=1}^{r} \hat{\phi}_{j}(u) h_{j}(u), h_{j} \in \widetilde{L}^{2}(0,2 \pi)\right\} \text {. }
$$

Clearly, $T_{n} V_{1}=V_{1}, n \in \mathbf{Z}$, and the following results are easy consequences.

Proposition 3.1. The following conditions are equivalent:

(1) $V_{0} \subset V_{1}$,

(2) $\left\{\phi_{1}, \ldots, \phi_{r}\right\} \subset V_{1}$,

(3) There exist $a_{i j} \in \widetilde{L}^{2}(0,2 \pi), i, j=1, \ldots, r$, such that

$$
\hat{\phi}_{i}(2 u)=\sum_{j=1}^{r} \hat{\phi}_{j}(u) a_{i j}(u), \quad i=1, \ldots, r .
$$

We shall henceforth assume that the conditions in Proposition 3.1 hold.

Proposition 3.2. Suppose that $f_{i} \in V_{1}, i=1, \ldots, r$, and

$$
\hat{f}_{i}(2 u)=\sum_{j=1}^{r} \hat{\phi}_{j}(u) h_{i j}(u), \quad h_{i j} \in \widetilde{L}^{2}(0,2 \pi), i, j=1, \ldots, r .
$$

Let $H:=\left(h_{i j}\right)_{i, j=1}^{r}$. Then $\left\{T_{n} f_{i}: n \in \mathbf{Z}, i=1, \ldots, r\right\}$ is orthonormal if and only if

$$
H(u) H(u)^{*}+H(u+\pi) H(u+\pi)^{*}=I_{r},
$$

where $I_{r}$ denotes the identity matrix of order $r$.

Proof. For $i, j=1, \ldots, r$,

$$
\begin{aligned}
\sum_{n \in \mathbf{Z}} \hat{f}_{i}(u+2 \pi n) \overline{\hat{f}_{j}(u+2 \pi n)} \\
=\sum_{k=1}^{r} \sum_{l=1}^{r} h_{i k}\left(\frac{u}{2}\right) \overline{h_{j l}\left(\frac{u}{2}\right)} \sum_{n \in \mathbf{Z}} \hat{\phi}_{k}\left(\frac{u}{2}+2 \pi n\right) \overline{\hat{\phi}_{l}\left(\frac{u}{2}+2 \pi n\right)} \\
\quad+\sum_{k=1}^{r} \sum_{l=1}^{r} h_{i k}\left(\frac{u}{2}+\pi\right) \overline{h_{j l}\left(\frac{u}{2}+\pi\right)} \sum_{n \in \mathbf{Z}} \hat{\phi}_{k}\left(\frac{u}{2}+\pi+2 \pi n\right) \overline{\hat{\phi}_{l}\left(\frac{u}{2}+\pi+2 \pi n\right)} \\
=\sum_{k=1}^{r} h_{i k}\left(\frac{u}{2}\right) \overline{h_{j k}\left(\frac{u}{2}\right)}+\sum_{k=1}^{r} h_{i k}\left(\frac{u}{2}+\pi\right) \overline{h_{j k}\left(\frac{u}{2}+\pi\right)},
\end{aligned}
$$

by Theorem 3.1 since $\left\{T_{n} \phi_{j}: n \in \mathbf{Z}, j=1, \ldots, r\right\}$ in an orthonormal set.

Therefore, $\left\{T_{n} f_{i}: n \in \mathbf{Z}, i=1, \ldots, r\right\}$ is orthonormal if and only if

$$
\sum_{n \in \mathbf{Z}} \hat{f}_{i}(u+2 \pi) \overline{\hat{f}_{j}(u+2 \pi)}=\delta_{i j}, \quad i, j=1, \ldots, r,
$$

if and only if

$$
\begin{aligned}
\sum_{k=1}^{r} h_{i k}\left(\frac{u}{2}\right) \overline{h_{j k}\left(\frac{u}{2}\right)}+\sum_{k=1}^{r} h_{i k}\left(\frac{u}{2}+\pi\right) \overline{h_{j k}\left(\frac{u}{2}+\pi\right)} & =\delta_{i j}, \\
i, j & =1, \ldots, r, u \in \mathbf{R} .
\end{aligned}
$$


Let $a_{i j}$ be defined as in (3.22), and $A:=\left(a_{i j}\right)_{i, j=1}^{r}$. By Proposition 3.2,

$$
A(u) A(u)^{*}+A(u+\pi) A(u+\pi)^{*}=I_{r} .
$$

Let $W_{0}$ be the orthogonal complement of $V_{0}$ in $V_{1}$, and let $\psi_{i} \in V_{1}, i=$ $1, \ldots, r$, such that

$$
\hat{\psi}_{i}(2 u)=\sum_{j=1}^{r} \hat{\phi}_{j}(u) b_{i j}(u)
$$

where $b_{i j} \in \widetilde{L}^{2}(0,2 \pi), i, j=1, \ldots, r$. Let $B:=\left(b_{i j}\right)_{i, j=1}^{r}$. A similar argument as in the proof of Proposition 3.2 gives

Proposition 3.3. The functions $\psi_{i}, i=1, \ldots, r$, are in $W_{0}$ if and only if

$$
A(u) B(u)^{*}+A(u+\pi) B(u+\pi)^{*}=0 .
$$

Furthermore, $\left\{T_{n} \psi_{i}: n \in \mathbf{Z}, i=1, \ldots, r\right\}$ is an orthonormal set if and only if

$$
B(u) B(u)^{*}+B(u+\pi) B(u+\pi)^{*}=I_{r} .
$$

Given $A=\left(a_{i j}\right)_{i, j=1}^{r}$ satisfying (3.23), the functions $\hat{\psi}_{i}, i=1, \ldots, r$, can be obtained by constructing $B=\left(b_{i j}\right)_{i, j=1}^{r}$ so that (3.25) and (3.26) are satisfied. Indeed (3.23) is equivalent to the orthonormality of the vectors

$$
\left(a_{i 1}(u), \ldots, a_{i r}(u), a_{i 1}(u+\pi), \ldots, a_{i r}(u+\pi)\right), \quad i=1, \ldots, r,
$$

for $u \in[0, \pi)$. By Gram-Schmidt process they can be extended to an orthonormal basis of $\mathbf{C}^{2 r}$. Let the new vectors in the basis be denoted by

$$
\left(b_{i 1}(u), \ldots, b_{i r}(u), b_{i 1}(u+\pi), \ldots, b_{i r}(u+\pi)\right), \quad i=1, \ldots, r,
$$

and extend $b_{i j}, i, j=1, \ldots, r$, periodically with period $2 \pi$. Then $B:=$ $\left(b_{i j}\right)_{i, j=1}^{r}$ satisfies (3.25) and (3.26). Let $\psi_{i}$ be defined as in (3.24).

Thoerem 3.5. The set $\left\{T_{n} \psi_{i}: n \in \mathbf{Z}, 1 \leq i \leq r\right\}$ is an orthonormal basis of $W_{0}$. Furthermore,

$$
W_{0}=\left\{f \in L^{2}(\mathbf{R}): \hat{f}=\sum_{j=1}^{r} \hat{\psi}_{j} h_{j}, h_{j} \in \widetilde{L}^{2}(0,2 \pi)\right\} .
$$

Proof. By Proposition 3.3, $\left\{T_{n} \psi_{i}: n \in \mathbf{Z}, 1 \leq i \leq r\right\}$ is an orthonormal subset of $W_{0}$. We have only to show that it is complete.

Take $\psi \in W_{0}$ and orthogonal to $\left\{T_{n} \psi_{i}: n \in \mathbf{Z}, 1 \leq i \leq r\right\}$. Then there exist $g_{1}, \ldots, g_{r}$, in $\widetilde{L}^{2}(0,2 \pi)$ such that

$$
\hat{\psi}(2 u)=\sum_{j=1}^{r} \hat{\phi}_{j}(u) g_{j}(u),
$$

and by a similar argument as in the proof of Proposition 3.2,

$$
\sum_{k=1}^{r}\left(a_{i k}(u) \overline{g_{k}(u)}+a_{i k}(u+\pi) \overline{g_{k}(u+\pi)}\right)=0
$$

and

$$
\sum_{k=1}^{r}\left(b_{i k}(u) \overline{g_{k}(u)}+b_{i k}(u+\pi) \overline{g_{k}(u+\pi)}\right)=0
$$


for $i=1, \ldots, r$. This means that the vector

$$
\left(g_{1}(u), \ldots, g_{r}(u), g_{1}(u+\pi), \ldots, g_{r}(u+\pi)\right)
$$

is orthogonal to the orthonormal basis of $\mathbf{C}^{2 r}$. Therefore, all $g_{j}, j=1, \ldots, r$, and hence $\psi$ are zero. This implies that $\left\{T_{n} \psi_{i}: n \in \mathbf{Z}, 1 \leq i \leq r\right\}$ is an orthonormal basis of $W_{0}$.

Functions in $W_{0}$ can be written uniquely in the form

$$
f=\sum_{j=1}^{r} \sum_{n \in \mathbf{Z}} c_{j n} T_{n} \psi_{j}, \quad\left(c_{j n}\right)_{n \in \mathbf{Z}} \in l^{2}(\mathbf{Z}) .
$$

Taking Fourier transform leads to (3.27).

\section{Multiresolution APPROXimation GENERATED BY CARDINAL HERMITE $B$-SPLINES}

Let $n$ and $r$ be positive integers, $n$ even, such that $2 r \leq n$, and

$$
\mathscr{S}_{n}^{r}:=\left\{f \in C^{n-r-1}(\mathbf{R}):\left.f\right|_{[\nu, \nu+1)} \in \Pi_{n-1}, \nu \in \mathbf{Z}\right\},
$$

where $\Pi_{n-1}$ is the class of polynomials of degree $\leq n-1$. Functions in $\mathscr{S}_{n}^{r}$ are called cardinal Hermite splines of degree $n-1$. For $j=0, \ldots, r-1$, let

$$
\mathscr{S}_{n, j}^{r}:=\left\{f \in \mathscr{S}_{n}^{r}: f^{(k)}(\nu)=0, \nu \in \mathbf{Z}, k=0, \ldots, r-1, k \neq j\right\} .
$$

The space $\mathscr{S}_{n, j}^{r}$ has a basis consisting of integer translates of a function $N_{n, j}=$ $N_{n, j}^{r} \in \mathscr{S}_{n, j}^{r}, j=0, \ldots, r-1$, with minimal support $\left[-\frac{n}{2}-1+r, \frac{n}{2}+1-r\right]$, in the sense that every $f \in \mathscr{S}_{n, j}^{r}$ has a unique representation of the form

$$
f(x)=\sum_{\nu \in \mathbf{Z}} c_{\nu} N_{n, j}(x-\nu), \quad x \in \mathbf{R}
$$

(see $[18,8])$. The functions $N_{n, j}$ are called cardinal Hermite $B$-splines.

For computational purposes, the Fourier transforms $\widehat{N}_{n, j}$ of the cardinal Hermite $B$-splines are very useful. They can be expressed in closed forms with the help of the Hankel determinant $\left|H_{r}\left(a_{n}\right)\right|$ where $H_{r}\left(a_{n}\right)$ is the matrix of order $r$ whose $j$ th column is $\left(a_{n-j+1}, \ldots, a_{n-j-r+2}\right)^{T}$ (see [9]). Indeed if

$$
\alpha_{k}(u):=\sum_{\nu \in \mathbf{Z}}(u+2 \pi \nu)^{-k}, \quad k=2, \ldots, n,
$$

and $H_{r, j}\left(\alpha_{n}(u)\right)$ denotes the matrix obtained from $H_{r}\left(\alpha_{n}(u)\right)$ by replacing the $(j+1)$ th column by $\left(u^{n}, u^{n-1}, \ldots, u^{n-r+1}\right)^{T}, j=0, \ldots, r-1$, then

$$
\widehat{N}_{n, j}(u)=\left(2 \sin \frac{u}{2}\right)^{n}\left|H_{r, j}\left(\alpha_{n}(u)\right)\right| \text {. }
$$

For $j=0, \ldots, r-1, N_{n, j}$ are regular. Indeed, $\widehat{N}_{n, j}$ are continuous and $\widehat{N}_{n, j}(u)=O\left(u^{-n+r-1}\right)$ as $n \rightarrow \infty$. Therefore the map $K: l^{2}(\mathbf{Z})^{r} \rightarrow L^{2}(\mathbf{R})$ such that

$$
K(s):=\sum_{j=0}^{r-1} \sum_{\nu \in \mathbf{Z}} s_{j}(\nu) T^{\nu} N_{n, j},
$$

is well defined. 
Theorem 4.1. The map $K$ defined by (4.6) is an isomorphism of $l^{2}(\mathbf{Z})^{r}$ onto a subspace of $L^{2}(\mathbf{R})$.

Proof. Let $\widehat{N}(u):=\left(\widehat{N}_{n, i}(u+2 \pi j)\right)_{i=0, j \in \mathbf{Z}}^{r-1}$. Suppose that $0<u<2 \pi$. By a result of [10], $\left|H_{r}\left(\alpha_{n}(u)\right)\right| \neq 0$. Therefore, equation (4.5) can be expressed in the form

$$
\left(\widehat{N}_{n, i}(u)\right)_{i=0}^{r-1}=(2 \sin u / 2)^{n}\left|H_{r}\left(\alpha_{n}(u)\right)\right| H_{r}\left(\alpha_{n}(u)\right)^{-1}\left(u^{-n}, u^{-n+1}, \ldots, u^{-n+r-1}\right)^{T} .
$$

Since $H_{r}\left(\alpha_{n}(u)\right)$ is $2 \pi$-periodic, it follows that

$$
\begin{aligned}
& \left(\hat{N}_{n, i}(u+2 \pi j)\right)_{i=0, r=0}^{r-1, r-1} \\
& \quad=(2 \sin u / 2)^{n}\left|H_{r}\left(\alpha_{n}(u)\right)\right| H_{r}\left(\alpha_{n}(u)\right)^{-1}\left((u+2 \pi j)^{-n+i}\right)_{i=0, j=0}^{r-1, r-1} .
\end{aligned}
$$

Since the last matrix on the right of (4.7) is a Vandermondian, we see that both sides are nonsingular. In particular $\widehat{N}(u)$ is of rank $r$, if $0<u<2 \pi$.

For $u=0$, a straightforward computation shows that

$$
\left(\widehat{N}_{n, i}(2 \pi j)\right)_{i=0, j=1}^{r-1, r}=\left|H_{r-1}\left(\beta_{n-2}\right)\right| H_{r-1}\left(\beta_{n-2}\right)^{-1}\left((2 \pi j)^{-n+i}\right)_{i=1, j=1}^{r-1, r-1},
$$

where

$$
\beta_{k}:=\sum_{\nu \neq 0}(2 \pi \nu)^{-k} .
$$

A similar argument as above shows that $\widehat{N}(0)$ is of rank $r$. The result now follows from Theorem 3.2.

Let $\widetilde{\mathscr{S}}_{n}^{r}$ denote the range of $K$. This is a closed subspace of $L^{2}(\mathbf{R})$. Furthermore, $\widetilde{\mathscr{S}}_{n}^{r}=\mathscr{S}_{n}^{r} \cap L^{2}(\mathbf{R})$. Therefore, if $D f(x):=f(2 x)$,

$$
\overline{\bigcup_{m \in \mathbf{Z}} D^{m} \widetilde{\mathscr{S}}_{n}^{r}} \supseteq \overline{\bigcup_{m \in \mathbf{Z}} D^{m} \widetilde{\mathscr{S}_{n}^{1}}}=L^{2}(\mathbf{R})
$$

Also $\bigcap_{m \in \mathbf{Z}} D^{m} \widetilde{\mathscr{S}}_{n}^{r}=\{0\}$, since it comprises polynomials in $L^{2}(\mathbf{R})$.

Let $V_{m}:=D^{m} \widetilde{\mathscr{S}}_{n}^{r}, m \in \mathbf{Z}$. Then $\left(V_{m}\right)_{m \in \mathbf{Z}}$ is a multiresolution approximation of $L^{2}(\mathbf{R})$ generated by the cardinal Hermite $B$-splines $N_{n, j}, j=$ $0, \ldots, r-1$. We shall call $\left(V_{m}\right)_{m \in \mathbf{Z}}$, a Hermite spline multiresolution approximation of $L^{2}(\mathbf{R})$.

\section{COMPACtLy SUPPORTED HeRMite SPLINE WAVELETS FOR THE ORTHOGONAL COMPLEMENT}

Let $W_{0}$ be the orthogonal complement of $V_{0}$ in $V_{1}$, so that $W_{m}:=D^{m} W_{0}$ is the orthogonal complement of $V_{m}$ in $V_{m+1}, m \in \mathbf{Z}$. For convenience, we shall assume that the $B$-spline $N_{n, j}$ is supported on [0,n-2r+2]. This can be easily achieved by translation.

Take $j=0, \ldots, r-1$, and consider functions of the form

$$
f_{j}(x)=\sum_{\nu=0}^{m} c_{j} N_{2 n, j}(2 x-\nu), \quad \text { with } f_{j}^{(j)}(k)=0, k \in \mathbf{Z},
$$

where $m$ is a positive integer. Then $f_{j}^{(n)} \in V_{1}$ and integrating by parts, in the sense of distributions, shows that $f_{j}^{(n)}$ is orthogonal to $V_{0}$, and hence belongs 
to $W_{0}$. Such a function $f_{j}$ with minimal support is given by

$$
f_{j}(x)=\sum_{\nu=0}^{2 n-2 r}(-1)^{\nu} N_{2 n, j}^{(j)}(\nu+1) N_{2 n, j}(2 x-\nu) \text {. }
$$

For $j=0, \ldots, r-1$, let

$$
\widetilde{\mathscr{S}}_{2 n, j}^{r}:=\left\{f \in \widetilde{\mathscr{S}}_{2 n}^{r}: f^{(k)}(\nu)=0, \nu \in \mathbf{Z}, k=0, \ldots, r-1, k \neq j\right\} .
$$

Lemma 5.1. Let $f \in D \widetilde{\mathscr{S}}_{2 n, j}^{r}$ such that $f(x)$ decays exponentially as $|x| \rightarrow \infty$ and $f^{(j)}(\nu)=0, \nu \in \mathbf{Z}$. Then

$$
f(x)=-\sum_{\nu \in \mathbf{Z}} a_{\nu} f_{j}(x-\nu),
$$

where $\left(a_{\nu}\right)_{\nu \in \mathbf{Z}}$ decays exponentially as $|\nu| \rightarrow \infty$.

Proof. Since $f \in D \widetilde{\mathscr{S}}_{2 n, j}^{r}$,

$$
f(x)=\sum_{\nu \in \mathbf{Z}} c_{\nu} N_{2 n, j}(2 x-\nu),
$$

where $\left(c_{\nu}\right)_{\nu \in \mathbf{Z}}$ decays exponentially as $|\nu| \rightarrow \infty$, and the condition $f^{(j)}(\nu)=0$, $\nu \in \mathbf{Z}$, leads to

$$
\sum_{\nu \in \mathbf{Z}} c_{\nu} N_{2 n, j}^{(j)}(2 k-\nu)=0, \quad k \in \mathbf{Z}
$$

Let

$$
p(z):=\sum_{\nu \in \mathbf{Z}} N_{2 n, j}^{(j)}(\nu) z^{\nu}, \quad q(z):=\sum_{\nu \in \mathbf{Z}} c_{\nu} z^{\nu} .
$$

Equation (5.4) is equivalent to

$$
p(z) q(z)+p(-z) q(-z)=0, \quad \text { for all } z \text {. }
$$

Since $N_{2 n, j}$ has compact support, $p$ is a polynomial. If $p$ has zeros $\left\{z_{j}\right\}$, then it does not have zeros $\left\{-z_{j}\right\}$, and so $\left\{-z_{j}\right\}$ are zeros of $q$. We can therefore write

$$
q(z)=p(-z) r(z) .
$$

Then $p(z) p(-z) r(z)+p(-z) p(z) r(-z)=0$, which gives $r(z)=-r(-z)$. Therefore, we can write

$$
r(z)=\sum_{\nu \in \mathbf{Z}} a_{\nu} z^{2 \nu-1}
$$

where $\left(a_{\nu}\right)_{\nu \in \mathbf{Z}}$ has exponential decay as $|\nu| \rightarrow \infty$, by (5.5). Also by (5.5),

$$
c_{\nu}=\sum_{l \in \mathbf{Z}} a_{l} N_{2 n, j}^{(j)}(\nu-2 l+1)(-1)^{\nu+1}
$$

Substituting (5.6) into (5.3) and using (5.1) leads to (5.2).

Proposition 5.1. Let $g \in D \widetilde{\mathscr{S}}_{2 n}^{r}$ such that $g(x)$ decays exponentially as $|x| \rightarrow$ $\infty$, and $g^{(j)}(\nu)=0, \nu \in \mathbf{Z}, j=0, \ldots, r-1$. Then

$$
g(x)=\sum_{j=0}^{r-1} \sum_{\nu \in \mathbf{Z}} a_{j \nu} f_{j}(x-\nu),
$$

where $\left(a_{j \nu}\right)_{\nu \in \mathbf{Z}}$ decays exponentially as $|\nu| \rightarrow \infty$. 
Proof. Since $g \in D \widetilde{\mathscr{S}}_{2 n}^{r}, g=g_{0}+g_{1}+\cdots+g_{r-1}$, where $g_{j} \in D \widetilde{\mathscr{S}}_{2 n, j}^{r}, j=$ $0, \ldots, r-1$, with $g_{j}^{(j)}(\nu)=0, \nu \in \mathbf{Z}$. The result follows from the previous lemma.

For $j=0, \ldots, r-1$, let $\psi_{j}:=f_{j}^{(n)}$, where $f_{j}$ is given by (5.1). Then $\psi_{j} \in W_{0}$ and is supported on $[0,2 n-2 r+1]$.

Theorem 5.1. The set $\left\{T_{\nu} \psi_{j}: \nu \in \mathbf{Z}, j=0, \ldots, r-1\right\}$ is complete in $W_{0}$.

Proof. It is sufficient to show that for $k=0, \ldots, r-1$,

$$
N_{n, k}(2 x)=f(x)+\sum_{j=0}^{r-1} \sum_{\nu \in \mathbf{Z}} a_{j \nu} \psi_{j}(x-\nu),
$$

where $f \in V_{0}:=\widetilde{\mathscr{S}}_{n}^{r}$ has exponential decay as $|x| \rightarrow \infty$ and $\left(a_{j \nu}\right)_{\nu \in \mathbf{Z}}$ decays exponentially as $|\nu| \rightarrow \infty$.

Choose $M \in D \widetilde{\mathscr{S}}_{2 n}^{r}$ with

$$
M^{(n)}(x)=N_{n, k}(2 x) .
$$

Since $N_{n, k}(2 x)$ is supported on $\left[0, \frac{n}{2}-r+1\right], M$ may be taken as a polynomial of degree $n-1$ outside [0, $\left.\frac{n}{2}-r+1\right]$. It can therefore be written as $M=F+G$, where $G \in D \widetilde{\mathscr{S}}_{2 n}^{r}$ has compact support, and $F \in \widetilde{\mathscr{S}}_{2 n}^{r}$ equals a polynomial of degree $n-1$ outside a compact set. Now let $H$ be the unique function in $\widetilde{\mathscr{S}}_{2 n}^{r}$ such that

$$
H^{(j)}(\nu)=G^{(j)}(\nu), \quad j=0, \ldots, r-1, \nu \in \mathbf{Z} .
$$

Since $G$ has compact support, the theory of cardinal Hermite interpolation shows that $H(x)$ decays exponentially as $|x| \rightarrow \infty$. Let $R:=G-H$. Then $R \in$ $D \widetilde{\mathscr{S}}_{2 n}^{r}, R^{(j)}(\nu)=0, j=0, \ldots, r-1, \nu \in \mathbf{Z}$, and $R(x)$ decays exponentially as $|x| \rightarrow \infty$, and we can write $M=F+H+R$. By Proposition 5.1, $R$ can be expressed as

$$
R(x)=\sum_{j=0}^{r-1} \sum_{\nu \in \mathbf{Z}} a_{j \nu} f_{j}(x-j),
$$

where $\left(a_{j \nu}\right)_{\nu \in \mathbf{Z}}$ decays exponentially as $|\nu| \rightarrow \infty$. Also by (4.3)

$$
H(x)=\sum_{j=0}^{r-1} \sum_{\nu \in \mathbf{Z}} b_{j \nu} N_{2 n, j}(x-\nu),
$$

where $\left(b_{j \nu}\right)_{\nu \in \mathbf{Z}}$ decays exponentially as $|\nu| \rightarrow \infty$. By (5.9)

$$
\begin{aligned}
N_{n, k}(2 x) & =F^{(n)}(x)+H^{(n)}(x)+R^{(n)}(x) \\
& =F^{(n)}(x)+\sum_{j=0}^{r-1} \sum_{\nu \in \mathbf{Z}} b_{j \nu} N_{2 n, j}^{(n)}(x-\nu)+\sum_{j=0}^{r-1} \sum_{\nu \in \mathbf{Z}} a_{j \nu} \psi_{j}(x-\nu) .
\end{aligned}
$$

Since $F^{(n)} \in V_{0}$ has compact support, and $H^{(n)} \in V_{0}$ has exponential decay, it follows that $F^{(n)}+H^{(n)} \in V_{0}$ has exponential decay. 


\section{REFERENCES}

1. G. Battle, A block spin construction of ondelettes, Part I: Lemarié functions, Comm. Math. Phys. 110 (1987), 601-615.

2. G. Battle and P. Federbush, Ondelettes and phase cell cluster expansion; a vindication, Comm. Math. Phys. 109 (1987), 417-419.

3. Charles K. Chui and Jian-zhong Wang, A cardinal spline approach to wavelets, Proc. Amer. Math. Soc. 113 (1991), 785-793.

4. _ A general framework of compactly supported splines and wavelets, J. Approx. Theory 71 (1992).

5. I. Daubechies, Orthonormal bases of compactly supported wavelet, Comm. Pure Appl. Math. 41 (1988), 909-996.

6. P. R. Halmos, A Hilbert space problem book, 2nd ed., Springer-Verlag, 1982.

7. S. Jaffard and Y. Meyer, Bases d'ondelettes dans des ouverts de $R^{n}$, J. Math. Pures Appl. 68 (1989), 95-108.

8. S. L. Lee, B-splines for cardinal Hermite interpolation, Linear Algebra and Appl. 12 (1975), 269-280.

9. __ Fourier transforms of B-splines and fundamental splines for cardinal Hermite interpolations, Proc. Amer. Math. Soc. 57 (1976), 291-296.

10. S. L. Lee and A. Sharma, Cardinal lacunary interpolation by g-splines. I. The characteristic polynomials, J. Approximation Theory 16 (1976), 85-96.

11. P. G. Lemarie, Ondelettes a localisation exponentielles, J. Math. Pures Appl. 67 (1988), 227-236.

12. S. Mallat, Multiresolution approximations and wavelet orthonormal bases of $L^{2}(\mathbf{R})$, Trans. Amer. Math. Soc. 315 (1989), 69-87.

13. _ $\ldots$ theory for multiresolution signal decomposition: the wavelet representation, IEEE Trans. on Pattern Analysis and Machine Intelligence 2 (1989), 674-693.

14. __ Multifrequency channel decomposition of images and wavelet models, IEEE Trans. ASSP 37 (1989), 2091-2110.

15. Y. Meyer, Ondelettes et fonctions splines, Seminaire Equations aux Derivees Partielles, École Polytechnique, Paris, 1986.

16. __ Principle d'incertitude, bases hilbertiennes et algèbres d'opérateurs, Bourbaki seminar, \#662, 1985-1986.

17. J. B. Robertson, On wandering subspaces for unitary operators, Proc. Amer. Math. Soc. 16 (1965), 233-236.

18. I. J. Schoenberg, Cardinal spline interpolation, CBMS-NSF Series in Appl. Math., no. 12, SIAM, Philadelphia, Pa., 1973.

Department of Mathematics, Texas A \& M University, College Station, Texas 77843 Current address: Department of Mathematics and Computer Science, University of Dundee, Dundee, DDI 4HN, Scotland

E-mail address: tgoodman@mcs.dundee.ac.uk

Department of Mathematics, National University of Singapore, 10 Kent Ridge CresCENT, SINGAPORE 0511

E-mail address, S. L. Lee: matleesl@nusvm.bitnet

E-mail address, W. S. Tang: mattws@nusvm.bitnet 